\title{
PEG-liposomal oxaliplatin combined with nuclear factor- $\kappa$ B inhibitor (PDTC) induces apoptosis in human colorectal cancer cells
}

\author{
CHUANG YANG ${ }^{1,2}$ and ZHONG-XUE FU ${ }^{2}$
}

\begin{abstract}
${ }^{1}$ Department of General Surgery, The Third People's Hospital of Mianyang, Mianyang, Sichuan 621000; ${ }^{2}$ Department of Gastrointestinal Surgery, The First Affiliated Hospital, Chongqing Medical University, Chongqing 400016, P.R. China
\end{abstract}

Received May 2, 2014; Accepted July 3, 2014

DOI: $10.3892 / 0 r .2014 .3336$

\begin{abstract}
To achieve sufficient antitumor activity for colorectal carcinoma, optimization of the therapeutic regimen is of great importance. The aim of the present study was to investigate the effects of polyethylene glycol (PEG)-liposomal oxaliplatin (L-OHP) on the induction of apoptosis in human colorectal cancer SW480 cells and how the nuclear factor $-\kappa \mathrm{B}(\mathrm{NF}-\kappa \mathrm{B})$ pathway may contribute to mediating PEG-liposomal L-OHPinduced apoptosis. PEG-liposomal L-OHP was prepared and used to treat colorectal cancer SW480 cells. SW480 cell uptake of liposomes was observed by laser focus or SEM. Apoptosis was measured by flow cytometry (FCM) and terminal deoxynucleotidyl transferase-mediated dUTP nick end labelling (TUNEL) assay. Expression of NF- $\mathrm{NB}, \mathrm{Bcl}-2$, Bax and activated caspase-3 (P17) was examined by western blot analyses. The results indicated that PEG-liposomal L-OHP induced apoptosis. When pretreated with pyrrolidine dithiocarbamate (PDTC), PEG-liposomal L-OHP induced a significant apoptotic response. Moreover, apoptosis was associated with concentration of PDTC. Expression of protein p-P65, Bcl-2 was downregulated, but Bax and P17 were upregulated. These findings indicate that PEG-liposomal L-OHP enhances the anticancer potency of the chemotherapeutic agent. Moreover, $\mathrm{NF}-\kappa \mathrm{B}$ signaling pathways may contribute to mediating PEG-liposomal L-OHP-induced apoptosis.
\end{abstract}

\section{Introduction}

Colorectal cancer (CRC) is a common type of malignant tumor with a high rate of morbidity and mortality. Early detection of $\mathrm{CRC}$ results in radical resection of the tumor in the majority

Correspondence to: Professor Zhong-Xue Fu, Department of Gastrointestinal Surgery, The First Affiliated Hospital, Chongqing Medical University, Chongqing 400016, P.R. China

E-mail: fzx990521@126.com

Key words: apoptosis, colorectal cancer, liposomes, oxaliplatin, nuclear factor- $\mathrm{KB}$ of cases. However, radical resection is curative for only $\sim 50 \%$ of the patients who will present local recurrence or distant metastases (1). The survival rate of patients with metastatic CRC (mCRC) has significantly improved with applications of molecularly targeted drugs, such as oxaliplatin (L-OHP), and has led to a substantial improvement in the overall survival rate. Chemotherapy drugs may exist for pathological parts of non-specific selection and result in the decrease of curative effect and side-effects on the body $(2,3)$.

Liposomes, as carriers of chemotherapeutic agents, can alter the distribution of these agents within the body and decrease drug toxicity $(4,5)$. Therefore, drug-loaded liposomes offer a new approach to the treatment of CRC. The polyethylene glycol (PEG) modification increases the affinity of liposomes for cancer cells and thus increases the cellular uptake of drugs. Cellular uptake of PEG-liposomal L-OHP induces bioactive changes in cells, resulting in apoptosis.

The transcription factor nuclear factor $-\kappa \mathrm{B}(\mathrm{NF}-\kappa \mathrm{B})$ is a dimeric complex composed of members of the Rel protein family, which includes p65 (RelA), p105/p50, p100/p52, RelB, c-Rel and the viral oncoprotein (v-Rel) (6). In its classic form, $\mathrm{NF}-\kappa \mathrm{B}$ is a dimer that consists of the transcriptionally inactive p50 subunit and the p65/RelA (p65) subunit. NF- $\kappa \mathrm{B}$ is a transcription factor that participates in proliferation and metastasis (7-9). The abnormality of NF- $\kappa \mathrm{B}$ is closely associated with human malignant tumor and different molecular modification of tumor cells results in abnormal activation regulation of $\mathrm{NF}-\kappa \mathrm{B}$ which loses its inducibility, thus remaining at an activated state, leading to abnormal regulation of apoptosis, cell cycle, adherence and metastatic genes by NF- $\mathrm{B}(10,11)$. $\mathrm{NF}-\kappa \mathrm{B}$ participates in the transcription and regulation of multiple cell factors in the apoptotic pathway $(11,12)$. Pyrrolidine dithiocarbamate (PDTC), a selective NF- $\kappa$ B inhibitor, has been used more widely in experimental study as the inhibitor of $\mathrm{NF}-\kappa \mathrm{B}$ for the treatment of inflammatory diseases (13). Moreover, it has been reported that PDTC could induce apoptosis via inhibition of NF- $\mathrm{NB}$ activation $(14,15)$.

In the present study, we investigated the effect of PEG-liposomal L-OHP on apoptosis, as well as regulation of apoptotic cell death by NF- $\kappa \mathrm{B}$, and we prepared PEG modified L-OHP liposomes to treat SW480 cells of human colorectal cancer in an effort to study the apoptosis. 


\section{Materials and methods}

Reagents. Human colorectal cancer SW480 cells were provided by the Life Science Academy of Chongqing Medical University. L-OHP was purchased from Sigma Co. 1,2-Distearoyl-snglycero-3-phosphoethanolamine-N-[ma-leimide(polyethylene glycol)-2000] (DSPE-PEG2000) was obtained from Avanti Polar Lipids, Inc. DIOC18(3), 3,3'-dioctadecyloxacarbocyanine perchlorate $(\mathrm{DiO})$ was obtained from Vigorous Biotech Co., Ltd. Rabbit polyclonal anti- $\beta$-actin, goat anti-rabbit IgG and peroxidase conjugated secondary antibodies were obtained from Bioscience Co., USA. Rabbit polyclonal antibodies of Bcl-2 and Bax were purchased from Santa Cruz Biotechnology, Inc. Activated-caspase-3 (P17) antibodies were obtained from Bioworld Technology, Inc. The TUNEL kit was purchased from Promega. NF- $\kappa$ B inhibitor, ammonium PDTC, was purchased from Beyotime Institute of Biotechnology.

Cell culture, cell uptake of liposomes observed by laser focus or scanning electron microscopy (SEM). Dio-labeled PEG-liposomes and PEG-liposomal L-OHP were prepared, as previously described (16). Human colorectal cancer SW480 cells in the logarithmic growth phase were trypsinized and incubated on glass slides $\left(5 \times 10^{3} / \mathrm{slide}\right)$ in $1 \mathrm{ml}$ of RPMI-1640 medium containing $10 \%$ FBS and pre-incubated for $24 \mathrm{~h}$. After removal of the culture medium, $1 \mathrm{ml}$ of fresh medium containing the Dio-labeled PEG-liposomes $(2 \mu \mathrm{mol} / \mathrm{ml})$ was added, followed by incubation at $37^{\circ} \mathrm{C}$. At $8 \mathrm{~h}$ post-incubation, the cells were washed twice with cold phosphate-buffered saline (PBS) and fixed with freshly prepared $4 \%$ paraformaldehyde (dissolved in pH 7.4 PBS). The cellular uptake of Dio-labeled PEG-liposomes was determined by measuring fluorescence and by SEM.

Immunofluorescence and flow cytometry (FCM) evaluate cellular calcium ion. The cells were placed in wells of a 6-well plate and glass slides incubated for $24 \mathrm{~h}$, respectively. The cells were treated with PEG-liposomal L-OHP (containing $28 \mu \mathrm{g}$ / $\mathrm{ml}$ L-OHP), PEG-liposomal L-OHP + PDTC (10 $\mu \mathrm{mol} / \mathrm{l})$ or PEG-liposomal L-OHP + PDTC ( $20 \mu \mathrm{mol} / \mathrm{l})$ for $12 \mathrm{~h}$, with no treatment as a control. After treatment, the culture medium was removed and washed by PBS. The cell glass slides were fixed with freshly prepared $4 \%$ paraformaldehyde, incubated with calcium ion fluorescent probe and evaluated for laser focus. The cells in 6-well plates were trypsinized, collected and fixed with $70 \%$ ice-cold ethanol overnight at $4^{\circ} \mathrm{C}$. Cells were centrifuged, re-suspended in $400 \mu 11 \mathrm{X}$ binding buffer $\left(>1 \times 10^{6} / \mathrm{ml}\right)$ and incubated with calcium ion fluorescent probe (final concentration $5 \mathrm{nmol} / \mathrm{ml}$ ) in the dark for $1 \mathrm{~h}$. The cells were washed with PBS and resuspended in $200 \mu \mathrm{l}$, and analyzed using a BD FACSCalibur (BD Biosciences).

Tagged deoxynucleotide transferase labeling. SW480 cells $\left(5 \times 10^{3} /\right.$ slide) were incubated on glass slides for $24 \mathrm{~h}$, treated for $12 \mathrm{~h}$ with PEG-liposomal L-OHP, PEG-liposomal $\mathrm{L}-\mathrm{OHP}+\mathrm{PDTC}(10 \mu \mathrm{mol} / \mathrm{l})$ or PEG-liposomal L-OHP + PDTC (20 $\mu \mathrm{mol} / \mathrm{l})$ and fixed in freshly prepared $4 \%$ paraformaldehyde (PFA; dissolved in $\mathrm{pH} 7.4 \mathrm{PBS}$ ) for $30 \mathrm{~min}$ at room temperature and finally washed with PBS, as described previously (17). Cells treated with DNase I, which fragments the DNA, served as positive controls.

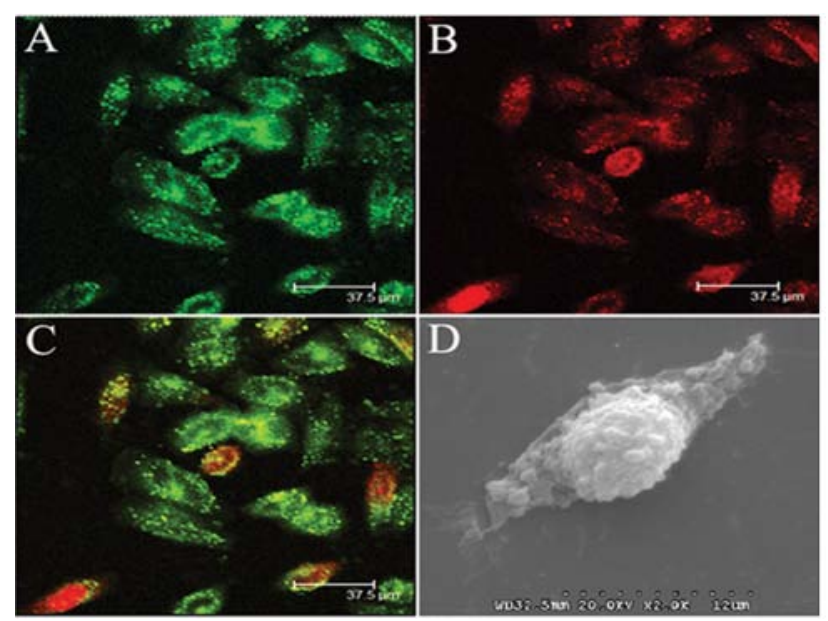

Figure 1. Cellular uptake of PEG-liposomes. Confocal microscopy showed that a considerable number of liposomes aggregated within cells $(\times 400)$. (A) Dio-labeled PEG-liposomes, green in color. (B) Cells were incubated with PI, which stained nuclei red. (C) A and B merge image. (D) PEGliposomes adhered to the cell surface, as detected by SEM.

Western blot analysis. The cells were collected and lysed and the protein concentration of each sample was determined using the Bradford dye-binding method. Protein samples $(50 \mu \mathrm{g})$ were electrophoresed on SDS/12 and 15\% PAGE gels and transferred to PVDF membranes (Millipore). The membranes were incubated overnight at $4^{\circ} \mathrm{C}$ with rabbit antibodies to Bcl-2, Bax, activated caspase-3 (1:500) and an anti- $\beta$-actin antibody (1:500), followed by incubation at room temperature for $1 \mathrm{~h}$ with a horseradish peroxidase (HRP)-labeled goat anti-rabbit secondary antibody added drop-wise concurrently with the enhanced chemiluminescence illuminating agent (Immobilon ${ }^{\mathrm{TM}}$ Western Chemiluminescent HRP Substrate, kit no. WBKLS0100). Finally, the membranes were visualized using a Bio-Rad system.

Statistical analyses. All values are expressed as means \pm SD using SPSS17.0 software. Experimental results were analyzed by the Student's t-test. $\mathrm{P}<0.05$ was considered as significant for values obtained for treated compounds compared with controls.

\section{Results}

Cellular uptake of liposomes. To study the conjugated effects of the cellular uptake of liposomes, confocal microscopy and scanning electron microscopy were adopted, respectively. Confocal microscopy demonstrated that after incubation in medium containing Dio-labeled liposomes for $4 \mathrm{~h}$, many of the PEG-liposomes conjugated with cells and aggregation of liposomes within cells (Fig. 1A-C). Also, scanning electron microscopy demonstrated that PEG-liposomes adhered to the cell surface (Fig. 1D).

Change of cellular calcium ion. Apoptosis signal transduction pathway in cells, calcium ions which activate apoptosis performer caspase-3, and hydrolysis of various cellular components lead to cell apoptosis. Cells were treated with PEG-liposomal L-OHP, and the change of calcium ion was 


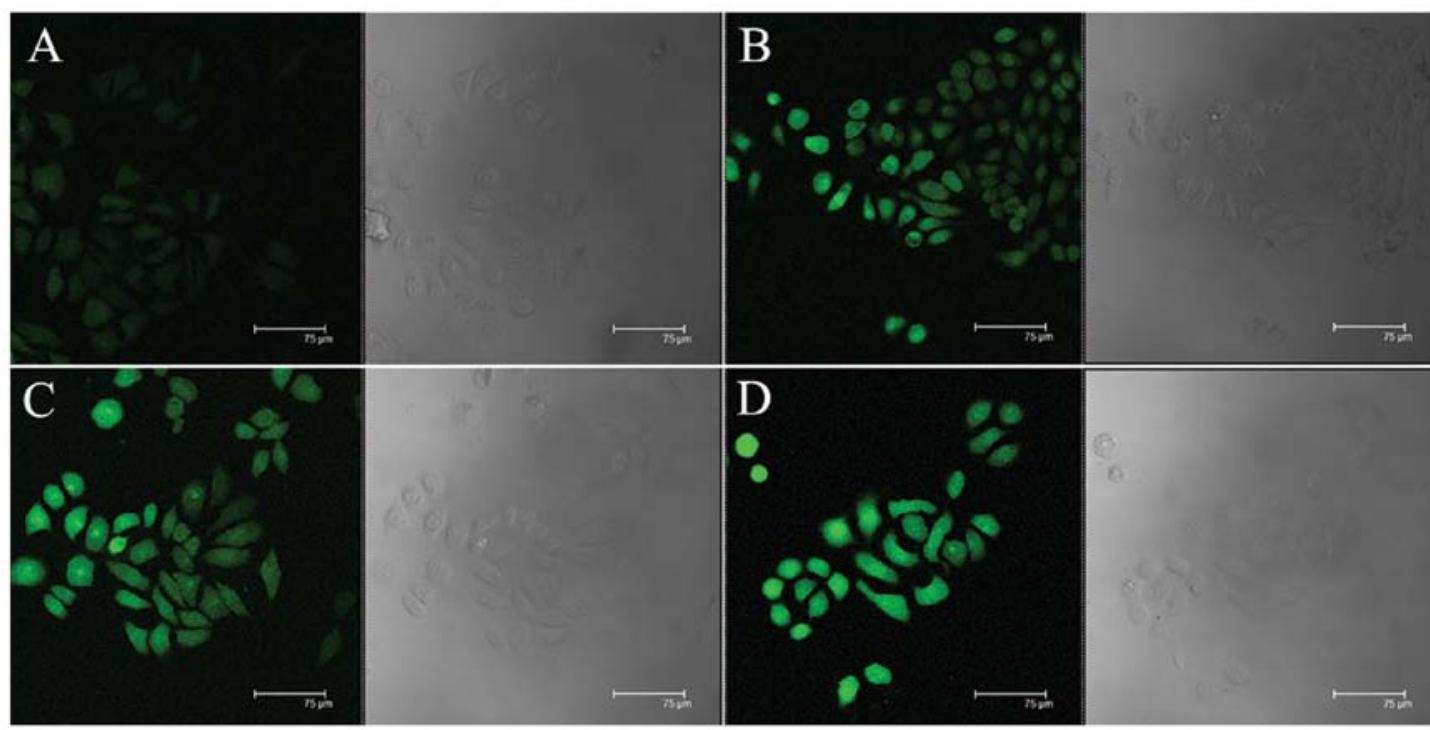

Figure 2. Cellular calcium ion evaluated by laser focus. Cells were incubated with calcium ion fluorescent probe and the cellular calcium ion was analyzed (A) Control. (B) PEG-liposomal L-OHP. (C) PEG-liposomal L-OHP + PDTC (10 $\mu \mathrm{mol} / 1)$. (D) PEG-liposomal L-OHP + PDTC (20 $\mu \mathrm{mol} / 1)$.
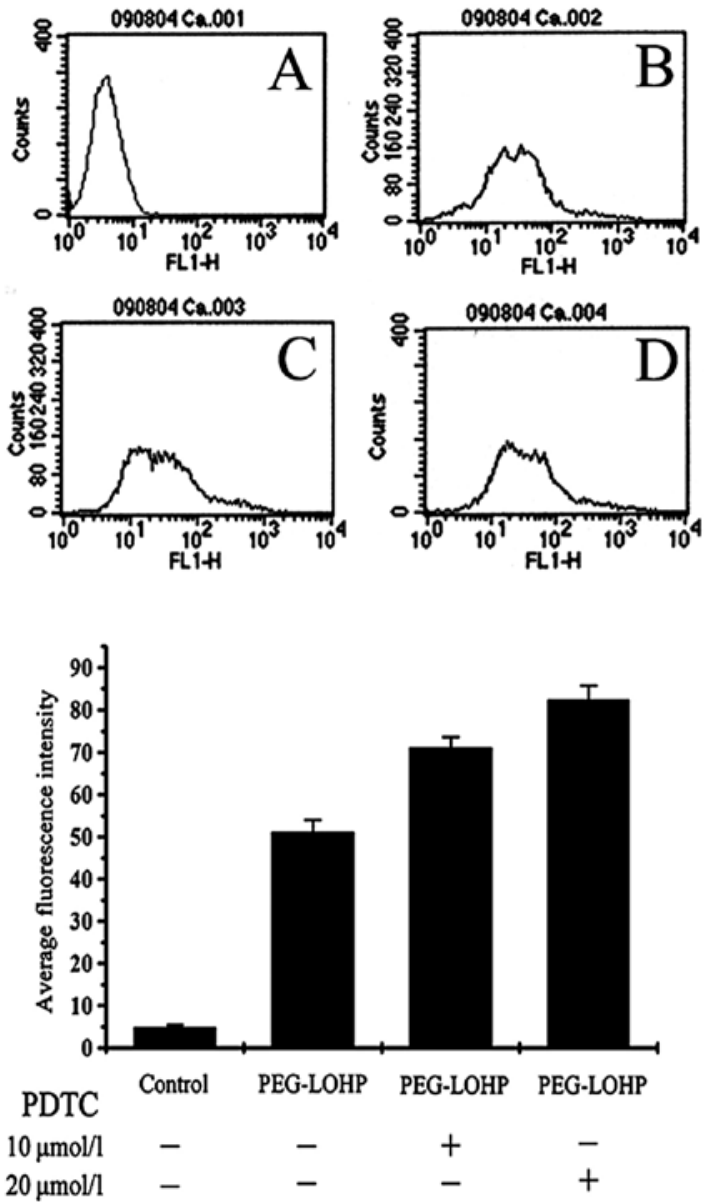

Figure 3. Cellular calcium ion evaluated by FCM. FCM demonstrated increased fluorescent intensity of cellular calcium ion, with increased concentration of PDTC.

obtained. Pretreatment with the NF-кB inhibitor, ammonium PDTC, demonstrated increased concentration of cellular calcium ion. The relative fluorescence intensity was $51.12 \pm 2.91$,

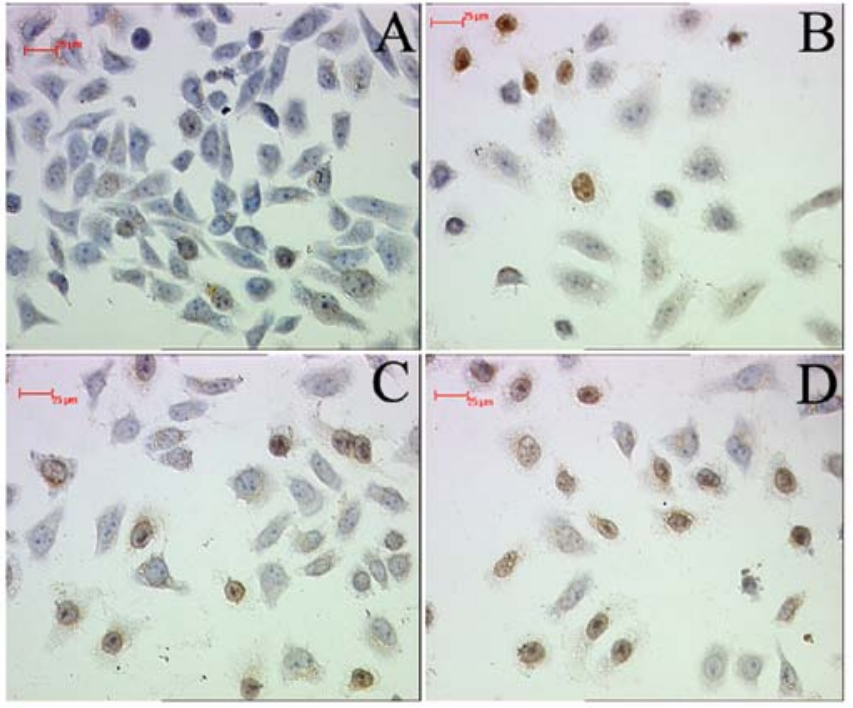

Figure 4. Apoptosis evaluated by TUNEL. After treatment, apoptosis was evaluated by TUNEL. The karyons assumed a yellowish brown color, with the staining of a karyon increasing as the level of apoptosis increased (DAB, x400 objective). (A) Control. (B) PEG-liposomal L-OHP. (C) PEGliposomal L-OHP + PDTC (10 $\mu \mathrm{mol} / \mathrm{l})$. (D) PEG-liposomal L-OHP + PDTC $(20 \mu \mathrm{mol} / 1)$.

$71.12 \pm 2.56$ and $82.44 \pm 3.27$, respectively, control each group, $\mathrm{P}<0.05$ (Figs. 2 and 3).

Apoptosis analysis. To establish the cytotoxic effect of PEG-liposomal L-OHP, apoptosis was assayed by TUNEL. The result demonstrated that PEG-liposomal L-OHP induced marked apoptotic death of SW480 cells, compared with control (Fig. 4B). However, pretreatment with PDTC led to strong detection of apoptosis in SW480 cells (Fig. 4C-D).

Expression protein of $N F-\kappa B(P 65)$. The phosphorylated NF-KB (p-P65) is able to translocate to the nucleus, activating genes 

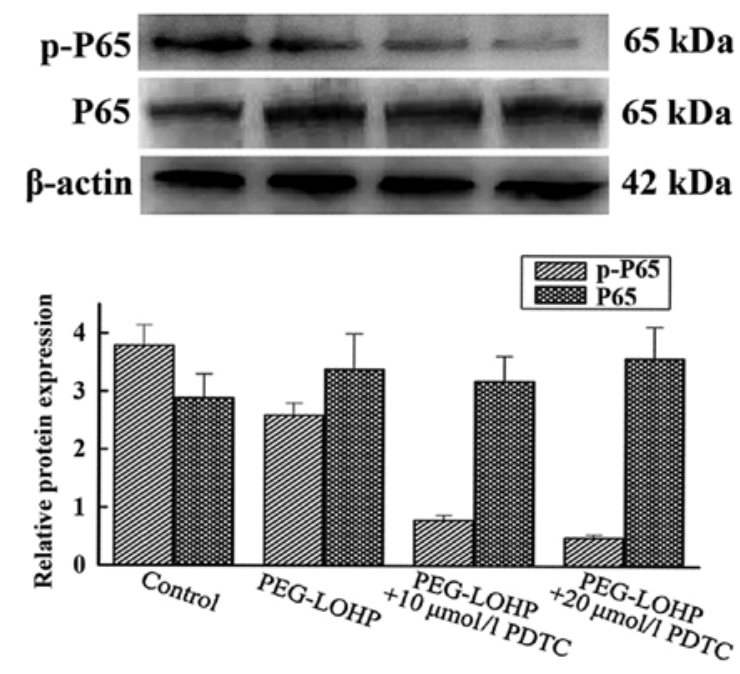

Figure 5.Expression of protein NF-кB. Cells were treated with PEG-liposoma L-OHP, PEG-liposomal L-OHP + PDTC $(10 \mu \mathrm{mol} / \mathrm{l})$ or PEG-liposomal L-OHP + PDTC $(20 \mu \mathrm{mol} / \mathrm{l})$, respectively. The total cells extract was prepared. The protein levels were determined by western blot analysis. Data are representative of three experiments (means $\pm \mathrm{SD}$ ).

involved in tumor cell proliferation and survival. To determine whether apoptosis is enhanced, after cells were pretreated with $\mathrm{NF}-\kappa \mathrm{B}$ inhibitor, total protein was extracted from the SW480 cells. The western blot results showed that protein of p-P65 was downregulated, compared with every other group, $\mathrm{P}<0.05$. However, the total protein of P65 was almost unchanged, compared with every other group, $\mathrm{P}>0.05$ (Fig. 5).

Expression of pro-apoptotic and anti-apoptotic proteins. Bcl-2, Bax, caspase-3 are associated with apoptosis, necrosis and autophagy and regulate all major types of cell death. To establish the cytotoxic effect of PEG-liposomal L-OHP, after pretreatment with various concentrations of NF- $\mathrm{KB}$ inhibitor, apoptosis was assayed by western blot analysis. The results showed Bcl-2 was downregulated. However, Bax and caspase-3 were upregulated, compared with every other group, $\mathrm{P}<0.05$ (Fig. 6).

\section{Discussion}

The nature of the active species generated in vivo, uptake, efflux, intracellular trafficking or insufficient diffusion in tumor tissues, lead to cytotoxic drugs having no target selectivity between normal body tissues and pathological sites (18), resulting in decreased curative effects and increased toxicity for certain chemotherapeutic agents.

The ideal therapy is to deliver the drugs directly to pathological sites. PEG-modified liposomes have been used as a carrier of anticancer drugs to enhance the affinity of anticancer drugs to cancer cells and uptake of anticancer drugs by cancer cells (19-21). Our in vitro study revealed uptake of PEG-liposomes by cancer cells at $2 \mathrm{~h}$, which increased with time of exposure and accumulation of a great amount of liposomes within the cells by fluorescence and SEM. The results are similar to those reported by Gabizon and Papahadjopoulos, and Gabizon $(22,23)$. Accordingly, FCM showed that compared with PEG-liposomal L-OHP, the same dosage of
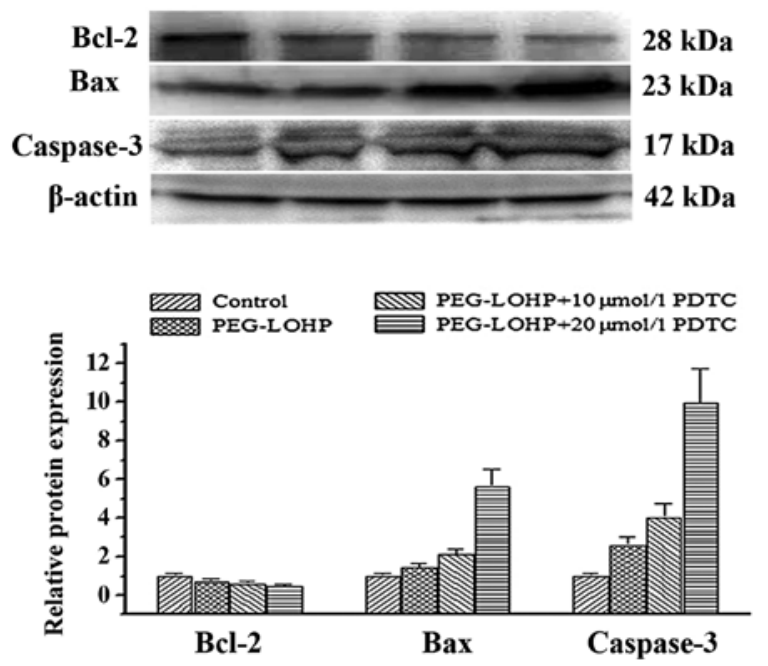

Figure 6. Expression of protein Bcl-2, Bax and caspase-3. Bcl-2 is an anti-apoptotic protein and guardian of the outer membrane and it preserves its integrity by opposing Bax. After treatment, the total cell extract was prepared, respectively. The protein levels were determined by western blot analysis. Data are representative of three experiments (means \pm SD).

PEG-liposomal L-OHP had a significantly greater impact on apoptotic cell death, after pretreatment with PDTC, the plasma $\mathrm{Ca}^{2+}$ level was consistently higher (Fig. 3). Moreover, we found that TUNEL experiments showed apoptotic death of SW480 cells was also consistently increased. PEG-liposomal L-OHP degraded in cells after pinocytosis; furthermore, the intracellular drug release enhanced active drug level within cells and slowed down drug efflux, therefore, liposome entrapment of chemotherapeutics represents its dual effect on tumor cells (24-26).

NF- $\mathrm{B}$, a complex of proteins, is a widespread transcription factor of eukaryotic cells with extensive actions and is involved in cellular inflammatory and apoptotic responses (27). $\mathrm{NF}-\kappa \mathrm{B}$ plays a key role in regulating apoptotic cell death. Our study showed that in the liposomal L-OHP-treated group, the apoptotic cells increased (Fig. 4B). To further explain the role of NF- $\mathrm{KB}$ in regulating apoptotic cell death, the tumor cells were administered PDTC pretreatment, which resulted in increased apoptotic cell death (Fig. 4C and D). However, the expression protein of NF- $\mathrm{KB}$ was markedly suppressed by PDTC pretreatment, P17, Bax and Bad showed an upward trend (Figs. 5 and 6). This may be explained by the selective $\mathrm{NF}-\kappa \mathrm{B}$ inhibition mechanism of PDTC.

Activated NF- $\kappa \mathrm{B}$ is highly expressed in tumor cells $(28,29)$; the decrease of the activity of NF- $\kappa B$ inhibits the expression of Bcl-2 and Bax $(30,31)$, predominantly seen in cytoplasm, and is highly expressed in many refractory tumors or those with poor prognosis such as gastric carcinoma and intestinal carcinoma.

Bcl-2 and Bax are members of the Bcl-2 family (32). They are associated with apoptosis, necrosis. Bcl-2 is an anti-apoptotic protein and guardian of the outer membrane. In contrast, Bax is a proapoptotic protein that induces mitochondrial outer membrane permeabilization, causing the release of caspase activating proteins and it preserves its integrity by opposing Bcl-2. The downregulation of Bcl-2 and upregulation of Bax and induced activation of caspase- 3 through upregulation 
of intracellular apoptotic pathway $(32,33)$, results in final apoptotic cell death $(34,35)$. In the present study, these results indicated that $\mathrm{NF}-\kappa \mathrm{B}$ plays a role in mediating regulation L-OHP PEG-liposome-induced aptoptotic cell death. PDTC was demonstrated to suppress the activation of NF- $\kappa \mathrm{B}$ and to ameliorate the cell death of SW480. Moreover, apoptosis was correlated with concentration of PDTC. Therefore, the results obtained in the present study indicate that the inhibition of $\mathrm{NF}-\kappa \mathrm{B}$ activation by PDTC in the PEG-liposomes L-OHP is likely to be associated with the blocking of $\mathrm{NF}-\kappa \mathrm{B}$ signal pathway, the downregulation of Bcl-2 expression and upregulation of Bax or caspase- 3 expression.

In conclusion, the experiments presented in this report indicate that PEG-liposome-targeting approaches represent a general framework for developing more effective cancer therapies. Liposome entrapment of L-OHP enhanced its anticancer potency. PDTC can enhance the cell apoptosis induced by PEG-liposomes. Inhibition of NF- $\kappa \mathrm{B}$ activation increases the antitumor effect of the antitumor agents. The dual treatment regimen is of great significance for the treatment of colorectal cancer. The success of specific combinations of targeted therapies may likely depend on the specificity and efficacy of each individual targeted therapy.

\section{Acknowledgements}

This study was supported by a grant from the Natural Science Foundation of China (no. 81172295). We thank the Institute of Life Science of Chongqing Medical University for their technical support and experimental assistance.

\section{References}

1. Spolverato G, Ejaz A, Azad N and Pawlik TM: Surgery for colorectal liver metastases: the evolution of determining prognosis. World J Gastrointest Oncol 5: 207-221, 2013.

2. Alberts SR, Sargent DJ, Nair S, et al: Effect of oxaliplatin, fluorouracil, and leucovorin with or without cetuximab on survival among patients with resected stage III colon cancer: a randomized trial. JAMA 307: 1383-1393, 2012.

3. Garcia-Foncillas J and Diaz-Rubio E: Progress in metastatic colorectal cancer: growing role of cetuximab to optimize clinical outcome. Clin Transl Oncol 12: 533-542, 2010.

4. Jain RL and Shastri JP: Study of ocular drug delivery system using drug-loaded liposomes. Int J Pharm Investig 1: 35-41, 2011

5. Saffari M, H Shirazi F, Oghabian MA, et al: Preparation and in-vitro evaluation of an antisense-containing cationic liposome against non-small cell lung cancer: a comparative preparation study. Iran J Pharm Res 12: 3-10, 2013.

6. Ashour AE, Abd-Allah AR, Korashy HM, et al: Thymoquinone suppression of the human hepatocellular carcinoma cell growth involves inhibition of IL-8 expression, elevated levels of TRAIL receptors, oxidative stress and apoptosis. Mol Cell Biochem 389: 85-98, 2014

7. Nookala AR, Shah A, Noel RJ and Kumar A: HIV-1 Tat-mediated induction of CCL5 in astrocytes involves NF- $\kappa \mathrm{B}, \mathrm{AP}-1, \mathrm{C} / \mathrm{EBP}$ and $\mathrm{C} / \mathrm{EBP} \gamma$ transcription factors and JAK, PI3K/Akt and p38 MAPK signaling pathways. PLoS One 8: e78855, 2013.

8. Zhang F, Lu M, Wang $\mathrm{H}$ and Ren T: Aspirin attenuates angiotensin II-induced inflammation in bone marrow mesenchymal stem cells via the inhibition of ERK1/2 and NF- $\kappa B$ activation. Biomed Rep 1: 930-934, 2013.

9. Mezzanotte L, An N, Mol IM, Löwik CW and Kaijzel EL: A new multicolor bioluminescence imaging platform to investigate $\mathrm{NF}-\kappa \mathrm{B}$ activity and apoptosis in human breast cancer cells. PLoS One 9: e85550, 2014.

10. Krizanova O, Markova J, Pacak K, et al: Triptolide induces apoptosis through the SERCA 3 upregulation in PC12 cells. Gen Physiol Biophys 33: 137-144, 2014.
11. Anttonen M, Pihlajoki M, Andersson N, et al: FOXL2, GATA4, and SMAD3 co-operatively modulate gene expression, cell viability and apoptosis in ovarian granulosa cell tumor cells. PLoS One 9: e85545, 2014.

12. Zhuang Y, Li D, Fu J, et al: Overexpression of AIOLOS inhibits cell proliferation and suppresses apoptosis in Nalm-6 cells. Oncol Rep 31: 1183-1190, 2014.

13. Huang Y, Lu Y, Zhang L, et al: Perineural dexmedetomidine attenuates inflammation in rat sciatic nerve via the NF- $\kappa \mathrm{B}$ pathway. Int J Mol Sci 15: 4049-4059, 2014.

14. Li T, Zhang Q, Zhang J, et al: Fenofibrate induces apoptosis of triple-negative breast cancer cells via activation of NF- $\kappa B$ pathway. BMC Cancer 14: 96, 2014.

15. Zhang J, Zhang DL, Jiao XL and Dong Q: S100A4 regulates migration and invasion in hepatocellular carcinoma HepG 2 cells via NF- $\kappa$ B-dependent MMP-9 signal. Eur Rev Med Pharmacol Sci 17: 2372-2382, 2013

16. Yang C, Liu HZ, Fu ZX and Lu WD: Oxaliplatin long-circulating liposomes improved therapeutic index of colorectal carcinoma. BMC Biotechnol 11: 21, 2011.

17. Yang C, Liu HZ and Fu ZX: Effects of PEG-liposomal oxaliplatin on apoptosis, and expression of Cyclin A and Cyclin D1 in colorectal cancer cells. Oncol Rep 28: 1006-1012, 2012.

18. Olszewski U and Hamilton G: A better platinum-based anticancer drug yet to come? Anticancer Agents Med Chem 10: 293-301, 2010.

19. Preiss MR and Bothun GD: Stimuli-responsive liposomenanoparticle assemblies. Expert Opin Drug Deliv 8: 1025-1040, 2011.

20. Suntres ZE: Liposomal antioxidants for protection against oxidant-induced damage. J Toxicol 2011: 152474, 2011.

21. Pagano RE and Weinstein JN: Interactions of liposomes with mammalian cells. Annu Rev Biophys Bioeng 7: 435-468, 1978.

22. Gabizon A and Papahadjopoulos D: Liposome formulations with prolonged circulation time in blood and enhanced uptake by tumors. Proc Natl Acad Sci USA 85: 6949-6953, 1988.

23. Gabizon AA: Stealth liposomes and tumor targeting: one step further in the quest for the magic bullet. Clin Cancer Res 7: 223-225, 2001

24. Noble GT, Stefanick JF, Ashley JD, et al: Ligand-targeted liposome design: challenges and fundamental considerations. Trends Biotechnol 32: 32-45, 2014

25. Hood RR, Shao C, Omiatek DM, et al: Microfluidic synthesis of PEG- and folate-conjugated liposomes for one-step formation of targeted stealth nanocarriers. Pharm Res 30: 1597-1607, 2013.

26. Abu Lila AS, Doi Y, Nakamura K, et al: Sequential administration with oxaliplatin-containing PEG-coated cationic liposomes promotes a significant delivery of subsequent dose into murine solid tumor. J Control Release 142: 167-173, 2010.

27. Vaiopoulos AG, Athanasoula KCh and Papavassiliou AG: NF-кB in colorectal cancer. J Mol Med 91: 1029-1037, 2013.

28. Li J, Li J, Yue Y, et al: Genistein suppresses tumor necrosis factor $\alpha$-induced inflammation via modulating reactive oxygen species/Akt/nuclear factor $\kappa \mathrm{B}$ and adenosine monophosphateactivated protein kinase signal pathways in human synoviocyte MH7A cells. Drug Des Devel Ther 8: 315-323, 2014

29. Zha Y, Gan P, Yao Q, Ran FM and Tan J: Downregulation of Rap1 promotes 5-fluorouracil-induced apoptosis in hepatocellular carcinoma cell line HepG2. Oncol Rep 31: 1691-1698, 2014.

30. Sethi G, Ahn KS, Sung B and Aggarwal BB: Pinitol targets nuclear factor-kappaB activation pathway leading to inhibition of gene products associated with proliferation, apoptosis, invasion, and angiogenesis. Mol Cancer Ther 7: 1604-1614, 2008.

31. Aggarwal BB: Nuclear factor-kappaB: the enemy within. Cancer Cell 6: 203-208, 2004

32. Fan XX, Li N, Wu JL, et al: Celastrol induces apoptosis in gefitinib-resistant non-small cell lung cancer cells via caspasesdependent pathways and Hsp90 client protein degradation. Molecules 19: 3508-3522, 2014.

33. Park MY, Jeong YJ, Kang GC, et al: Nitric oxide-induced apoptosis of human dental pulp cells is mediated by the mitochondria-dependent pathway. Korean J Physiol Pharmacol 18: 25-32, 2014.

34. Kim MH, Kim SH and Yang WM: Beneficial effects of Astragaloside IV for hair loss via inhibition of Fas/Fas L-mediated apoptotic signaling. PLoS One 9: e92984, 2014.

35. Liu G, Wang T, Wang T, Song J and Zhou Z: Effects of apoptosis-related proteins caspase-3, Bax and Bcl-2 on cerebral ischemia rats. Biomed Rep 1: 861-867, 2013. 